\title{
Urinalysis: Extract the Relevant Information Before Throwing it into the Drain
}

\author{
Lamsal Madhab ${ }^{1}$
}

\section{Affiliations:}

${ }^{1}$ Department of Biochemistry, BP Koirala Institute of Health Sciences, Dharan, Nepal

Correspondence to:

Madhab Lamsal,

Professor, Department of Biochemistry

BP Koirala Institute of Health Sciences

Dharan, Nepal

madhab.lamsal@bpkihs.edu

How to cite this Article:

Lamsal M. Urinalysis: Extract the Relevant Information Before Throwing it into the Drain. Ann. Clin. Chem. Lab. Med. 2021:4(1);1-3

DOI: https://doi.org/10.3126/acclm.v4i1.42672

(C) 2022 Nepalese Association for Clinical Chemistry

This work is licensed under a Creative Commons Attribution-Share Alike 4.0 International License.
From ancient time urine has been considered as a substance of importance and examination for physical wellbeing. Evidences from the ancient civilizations including the Egyptian, Sumerian, Babylonian and Eastern Civilizations such as Vedic cultures support the use of urine as an index of physical and mental wellbeing. Classified as coloured, black, frothy, cloudy and sweet, urine used to be correlated with different disease conditions such as jaundice, kidney diseases, diabetes etc. These practices have been carried on even by the alchemists and have now formed as an integral constituent of clinical laboratory diagnostics. $^{1,2}$

Modern approach to urinalysis can be credited to Dr. Richard Bright, MD, who in 1827 by performing urine examinations related to volume, colour, $\mathrm{pH}$, protein (but not cast) correlated his findings to several diseases and clinical picture including edema, proteinuria etc. Dr. Golding bird (1846) included the study of sediments in addition to above parameters as urinalysis. $^{1,2}$

Urinalysis combines the expertise from various disciplines including biochemistry, pathology, microbiology, cytology etc. Urinalysis may be used for screening, diagnosis, monitoring and prognosis due to the ease of collection in any settings. Modern day techniques such as molecular biology, immunology, and mass spectrometry with high resolution microscopy have taken up urinalysis to explore the genetic predisposition to inherited diseases and tumor studies besides the routine diagnostics. So, variation occurs in urinalysis from the simple routine analysis, microscopic examination to highly sophisticated and advanced automated analytical techniques. Urinalysis therefore, forms a key component of personalized medicine also integrating with proteomics, genomics, metabolomics approaches. $^{3}$ 
According to the National Committee for Clinical Laboratory Standard (NCCLS), urine specimen are classified into: patient collection, supervised collection and assisted collection. Depending on the clinical situation and purpose the urine collection procedure varies. While minimal instruction will be sufficient to collect urine from cooperative patients and obtain either as random, timed, or 24 hours collection, strict supervision is required if the urine collection is required for microbiological analysis and culture for which midstream urine is recommended. Proper hygiene and cleanliness of urethra (female) or glans penis (male) is mandatory to collect urine specimen for proper investigation. Assisted collection through participation of trained health professional is required to collect catheter and suprapubic specimen, following aseptic technique. Collection of suitable urine from pediatric patients and old aged on diapers is also a challenge. ${ }^{4}$

Often received urine specimens are unsuitable for analysis or culture due to pre-analytical error related to the method of collection, unsuitable containers, time and place besides the lack of knowledge in storage and transportation. Hence, very important information related to the metabolic status of the patient is lost. While urine collection and issues related to the preanalytical error is challenging, the methodologies and approaches during analysis is equally important and often neglected. The quality control issues related to urinalysis remains even more challenging in many diagnostic laboratories. In this respect there should be proper coordination and knowledge sharing among the physicians, patients, health professionals, care takers and technical team.

Urine collection from normal healthy subjects as volunteer is much more challenging than the patients needing those tests. Urinalysis of the patient admitted in the emergency ward is often delayed in comparison to the blood chemistry report, thereby increasing the holding time in emergency ward. Use of rapid test kits or POCT devices are therefore appropriate in such settings. The need of rapid analysis of various metabolites in urine determines the urgency of medical investigations eg. in diabetic ketogenesis, pregnancy testing, drug abuse etc. Nevertheless, considering the importance of urinalysis in clinical settings, more programmatic approaches should be adopted to derive maximum information from it before it is thrown into the drain.

At some point of time each drop of urine is equally important to the drop of blood. The volume, consistency, smell, colour etc. still carry significance in diagnosis and therefore should not be neglected at all. Hence, urine sample should be properly handled or properly processed to avoid eg. loss of glucose, ketone bodies, bilirubin etc or increasing the interferences due to bacterial growth.

Modern equipment dedicated for urinalysis combines the microscopic and chemistry together. Use of mass spectroscopy has further shown better prospects in urinalysis facilitating the metabolomics studies to integrate into patient care services. ${ }^{1-3,6}$ The use of urine cast analysis or urine as a source of DNA or circulating tumor cells correlating to the differential diagnosis of disease has been given due importance in recent years but as these needed automation and more advanced technologies, such facilities are lacking in most developing and under developed countries.

With the modernization in clinical settings, automation has been taking up urinalysis into wider arena of clinical practice. Albeit slowly, even in developing and under developed countries urinalysis is gaining its importance in diagnosis and prognosis of several diseases due to its easier and none to minimal invasive collection procedure. Nevertheless, considering its due importance in laboratory diagnostics, urinalysis should be done correctly and systematically with the incorporation of quality control for the overall benefit of the patients. If neglected and missed proper channel in collection and transportation, urinalysis may be misinterpreted and may also lead to serious medico legal issues. Therefore, like any other body fluids and tissues for laboratory analysis, Urinalysis should be treated as an important tool in clinical practice involving due ethical considerations. Therefore, urinalysis can be considered as a perfect science if done correctly and with quality control and not a substance to just pass into the drain.

\section{REFERENCES}

1. Brunzel NA. Fundamentals of Urine \& Body Fluid Analysis. WB Saunders ed., Philadelphia, 1994. 
2. National Committee for Clinical Laboratory Standard. Urinalysis and Collection, Transportation and Preservation of Urine Specimens: Approved Guideline NCCLS Document GP 16-A Vol 15, No15. Wayne, PA NCCLS, December,1995.

3. McBride LJ . Textbook of Urinalysis and Body Fluids: A Clinical Approach. Lippincott Williams and Wilkins Pub, Philadelphia, PA,1998.

4. Bishop ML. Fody EP, Schoeff LE. Clinical Chemistry Techniques, Principles, Correlations. $6^{\text {th }}$ edition Walters Kluwer, Lippincott Williams \& Wilkins, Philadelphia PA 2010.

5. Mundt LA, Shanahan K. Graff's Textbook of Routine Urinalysis and Body Fluids. Lippincott Williams \& Wilkins, 2010.

6. Strasinger SK, DiLirenzo MS. Urinalysis and Body Fluids. $6^{\text {th }}$ edition, Philadelphia: FA Davis Co: 2014. 